\title{
Céphalées tardives après ponction rachidienne avec une aiguille de Withacre 25G
}

\section{Delayed postdural puncture headache with a 25G Whitacre needle}

\author{
Jean-Christian Sleth, MD
}

Received: 22 June 2009/Accepted: 9 July 2009/Published online: 28 July 2009

(C) Canadian Anesthesiologists' Society 2009

\section{Au rédacteur en chef,}

Les céphalées après ponction durale sont habituellement de survenue précoce. Toutefois, un cas de céphalées apparaissant 10 jours après une péridurale en obstétrique a été récemment décrit. ${ }^{1}$ Nous souhaitons rapporter, avec l'accord de l'intéressée, un cas de céphalées de survenue tardive après rachianesthésie.

La patiente est une chirurgienne de 28 ans, à sa première grossesse, qui est hospitalisée pour une césarienne programmée sous rachianesthésie. La ponction est effectuée au niveau L4-L5 sans difficulté avec une aiguille 25G Withacre (Laboratoires Vygon, France). On injecte alors de la bupivacaïne hyperbare $10 \mathrm{mg}$, de la morphine $100 \mu \mathrm{g}$ et du sufentanil 2,5 $\mu \mathrm{g}$. Les suites sont simples hormis quelques nausées postopératoires imputées aux morphiniques. La patiente retourne chez elle après six jours. Au 8e jour apparaissent quelques céphalées qu'elle impute alors à la prise de bromocriptine et aux modifications hormonales du postpartum. Au 10e jour, la patiente constate des nausées associées à quelques vomissements. Les céphalées s'intensifient en fréquence et en intensité, elles sont diffuses et invalidantes de type « pique à glace » et un certain degré de photophobie est associé. Au 15e jour, la patiente reconnait le caractère positionnel de ces céphalées. Elle évoque alors la possibilité d'une relation entre ses symptômes et la rachianesthésie et prend contact avec nous au $17 \mathrm{e}$ jour. Après confirmation de l'absence d'anomalie au scanner cérébral, une péridurale est réalisée au niveau L3-L4 pour l'injection de $15 \mathrm{ml}$ de sang autologue, ce qui

J.-C. Sleth, MD ( $ه)$

Polyclinique Saint-Roch, Montpellier, France

e-mail: jean-christian.sleth@wanadoo.fr apporte une disparition des symptômes après 20 minutes de décubitus dorsal. Elle sort de l'hôpital le lendemain et l'évolution est favorable sans récidive avec un recul de 2 mois.

Selon une méta-analyse qui fait désormais autorité, les céphalées post rachianesthésie observées en obstétrique après ponction avec des aiguilles de type pointe crayon surviennent habituellement à la 48e heure et jusqu'au septième jour et durent d'un à sept jours. ${ }^{2}$

Cependant l'auteur de cette méta-analyse reconnaît que bien que très nombreuses, les données accumulées sur ces céphalées sont peu homogènes et rendent des conclusions délicates. ${ }^{3}$ Parmi les 22 études randomisées et contrôlées analysées, seule une a un score de 5 sur l'échelle de Jadad, contre 8 qui ont un score de 1. La qualité de ces études est donc en général faible et on n'a donc pas de réponses définitives en ce qui concerne la date de début des symptômes, leur intensité et leur évolution. De plus, la plupart des études limitent la durée de surveillance à la période d'hospitalisation; seule une étude assure un suivi sur une période prolongée de 6 semaines. ${ }^{4}$ Il n'est donc pas exclu que des formes tardives soient ignorées avec un défaut de prise en charge et un risque d'évolution vers la chronicisation, comme cela a déjà été décrit pour l'anesthésie péridurale après brèche durale négligée.

Il nous semble donc que le meilleur garant d'une prise en charge efficace passe par une amélioration de l'information non seulement des patientes mais aussi des médecins généralistes, ${ }^{5}$ gynécologues et sages-femmes afin qu'ils connaissent les caractéristiques d'une céphalée attribuable à une ponction de la dure-mère. Ceci permettrait d'orienter sans délai les patientes vers l'équipe anesthésique pour établir le diagnostic et le traitement. Le diagnostic ne doit pas être éliminé même si les symptômes surviennent plus d'une semaine après l'anesthésie rachidienne. 


\section{Conflit d'intérêt Aucun}

\section{Références}

1. Reamy BV. Post-epidural headache: how late can it occur? J Am Board Fam Med 2009; 22: 202-5.

2. Choi PT, Galinski SE, Takeuchi L, Lucas S, Tamayo C, Jadad AR. $\mathrm{PDPH}$ is a common complication of neuraxial blockade in parturients: a meta-analysis of obstetrical studies. Can J Anesth 2003; 50: $460-9$.
3. Wong AW. Is PDPH from a 25-gauge Whitacre needle always short-lasting and self-resolving? (author reply). Can J Anesth. 2004; 51: 637-8.

4. Hopkinson JM, Samaan AK, Russell IF, Birks RJ, Patrick MR. A comparative multicentre trial of spinal needles for caesarean section. Anaesthesia 1997; 52: 1005-11.

5. Duggan T, Simpson A. Use of an information leaflet to improve general practitioners' knowledge of post dural puncture headache. Int J Obstet Anesth 2008; 17: 82. 